\title{
EXTREMAL GRAPHS WITHOUT TOPOLOGICAL COMPLETE SUBGRAPHS*
}

\author{
M. CERA ${ }^{\dagger}$, A. DIÁNEZ ${ }^{\ddagger}$ AND A. MÁRQUEZ ${ }^{\S}$
}

\begin{abstract}
The exact values of the function $\operatorname{ex}\left(n ; T K_{p}\right)$ are known for $\left\lceil\frac{2 n+5}{3}\right\rceil \leq p<n$ (see [Cera, Diánez, and Márquez, SIAM J. Discrete Math., 13 (2000), pp. 295-301]), where ex (n; TK $\left.K_{p}\right)$ is the maximum number of edges of a graph of order $n$ not containing a subgraph homeomorphic to the complete graph of order $p$. In this paper, for $\left\lceil\frac{2 n+6}{3}\right\rceil \leq p<n-3$, we characterize the family of extremal graphs $\operatorname{EX}\left(n ; T K_{p}\right)$, i.e., the family of graphs with $n$ vertices and $e x\left(n ; T K_{p}\right)$ edges not containing a subgraph homeomorphic to the complete graph of order $p$.
\end{abstract}

Key words. extremal graph theory, topological complete subgraphs

AMS subject classifications. $05 \mathrm{C} 35,05 \mathrm{C} 70$

DOI. $10.1137 /$ S0895480100378677

1. Introduction. The study of the function $\operatorname{ex}\left(n ; T K_{p}\right)$-i.e., the maximum number of edges of a graph of order $n$ not containing a subgraph homeomorphic to $K_{p}$, where $K_{p}$ is the complete graph with $p$ vertices - is one of the most general extremal problems, as pointed out by Bollobas in [1]. Exact values for this function are known only in some cases, as can be seen in Table 1.1.

TABLE 1.1

Exact values of the function ex $\left(n ; T K_{p}\right)$.

\begin{tabular}{|c|c|c|}
\hline$p$ & $e x\left(n ; T K_{p}\right)$ & Reference \\
\hline \hline 3 & $n-1$ & {$[3]$} \\
\hline 5 & $2 n-3$ & {$[4],[8],[9]$} \\
\hline$\vdots$ & $\vdots$ & $\vdots$ \\
\hline$\left\lceil\frac{2 n+5}{3}\right\rceil \leq p<\left\lceil\frac{3 n+2}{4}\right\rceil$ & $\left(\begin{array}{c}n \\
2\end{array}\right)-(5 n-6 p+3)$ & {$[2]$} \\
\hline$\left\lceil\frac{3 n+2}{4}\right\rceil \leq p<n$ & $\left(\begin{array}{c}n \\
2\end{array}\right)-(2 n-2 p+1)$ & {$[2]$} \\
\hline
\end{tabular}

The aim of this work is to characterize a family of extremal graphs $E X\left(n ; T K_{p}\right)$ for appropriate values of $n$ and $p$, i.e., the set of graphs of order $n$, with $\operatorname{ex}\left(n ; T K_{p}\right)$

*Received by the editors September 28, 2000; accepted for publication (in revised form) July 1, 2004; published electronically December 9, 2004. This research was partially supported by the Ministry of Science and Technology, Spain, Research Project BMF2001-2474.

http://www.siam.org/journals/sidma/18-2/37867.html

$\dagger$ E.U.I.T. Agrícola, Universidad de Sevilla, Ctra. Utrera s/n, 41013-Sevilla, Spain (mcera@us.es).

${ }^{\ddagger}$ E.T.S. Arquitectura, Universidad de Sevilla, Reina Mercedes 2, 41012-Sevilla, Spain (anadianez@ us.es).

$\S$ E.T.S.I. Informática, Universidad de Sevilla, Reina Mercedes s/n, 41012-Sevilla, Spain (almar@ us.es). 
edges and not containing any subgraph homeomorphic to $K_{p}$. Actually, we characterize the family $\operatorname{EX}\left(n ; T K_{p}\right)$ for $\left\lceil\frac{2 n+6}{3}\right\rceil \leq p<n-3$ :

$$
\operatorname{EX}\left(n ; T K_{p}\right)= \begin{cases}(3 n-4 p+2) \overline{K_{3}}+(6 p-4 n-3) \overline{K_{2}} & \text { for }\left\lceil\frac{2 n+6}{3}\right\rceil \leq p<\left\lceil\frac{3 n+2}{4}\right\rceil, \\ K_{4 p-3 n-2}+(2 n-2 p+1) \overline{K_{2}} & \text { for }\left\lceil\frac{3 n+2}{4}\right\rceil \leq p<n-3 .\end{cases}
$$

2. Definitions and notation. Given a graph $H$ and a set $\left\{v_{1}, \ldots, v_{q}\right\}$ of vertices of $H$, we denote by $H_{0}=H$ and by $H_{k}$ for $k=1, \ldots, q$ the induced subgraph in $H$ by the set of vertices $V(H)-\left\{v_{1}, \ldots, v_{k}\right\}$. We denote by $\Delta(H)$ the maximum degree of the graph $H$ and by $\delta_{H}(v)$ the degree of the vertex $v$ in the graph $H$. The complement graph of $H$ will be denoted by $\bar{H}$.

Let $q$ and $s$ be a pair of nonnegative integers; $\mathcal{C}_{q}^{s}$ denotes the set of graphs $H$ such that there exists a set $\left\{v_{1}, \ldots, v_{q}\right\}$ of vertices of $H$ verifying the following:

(1) $\delta_{H_{j-1}}\left(v_{j}\right) \geq \delta_{H_{j}}\left(v_{j+1}\right)$ for $j=1, \ldots, q-1$.

(2) For each positive integer $h$, if there exists $k \in\{1, \ldots, q\}$ and $v \in H_{k}$ such that $\delta_{H_{k}}(v) \geq h$, then $\delta_{H_{j}}\left(v_{j+1}\right) \geq h$ for all $j=1, \ldots, k$.

(3) $H_{q}$ has at most $s$ edges (i.e., $\left|E\left(H_{q}\right)\right| \leq s$ ).

The next results show different conditions to guarantee that a graph belongs to the family described above (see [2]).

Lemma 2.1 (see [2]). Let $H$ be a graph with $n$ vertices. Then, for any $q \leq n$, there exists s such that $H$ is in $\mathcal{C}_{q}^{s}$.

When $s=q$, we know sufficient conditions for the edges of a graph to belong to the class $\mathcal{C}_{q}^{q}$.

Lemma 2.2 (see [2]). Let $n$ and $q$ be two positive integers, with $q<n$. If $H$ is a graph with $n$ vertices and $2 q$ edges, then

1. $H \in \mathcal{C}_{q}^{q}$,

2. $\delta_{H_{q}}(v) \leq 1$ for $v \in V\left(H_{q}\right)$.

Lemma 2.3 (see [2]). Let $q$ and $k$ be two positive integers with $k \leq q-2$. Let $H$ be a graph with $4 q-k+1$ vertices and $2 q+k+1$ edges. Then $H \in \mathcal{C}_{q}^{q}$.

Notation and terminology not given here can be found in [1] and [2].

3. The family of extremal graphs. In this section, we will characterize the family $E X\left(n ; T K_{p}\right)$ for $\left\lceil\frac{2 n+6}{3}\right\rceil \leq p<n-3$. This problem is equivalent to characterizing $E X\left(n ; T K_{n-q}\right)$ for $n \geq 4 q+2$ with $q \geq 4$ (case $\left\lceil\frac{3 n+2}{4}\right\rceil \leq p<n-3$ ) and $n=4 q-k+1$ with $q \geq 5,0 \leq k \leq q-5$ (the case $\left\lceil\frac{2 n+6}{3}\right\rceil \leq p<\left\lceil\frac{3 n+2}{4}\right\rceil$ ).

In order to avoid excessive repetition, we define the graphs $\mathcal{H}\left(n ; T K_{n-q}\right)$ :

$\mathcal{H}\left(n ; T K_{n-q}\right)= \begin{cases}K_{n-(4 q+2)}+(2 q+1) \overline{K_{2}} & \text { for } n \geq 4 q+2, \\ (k+1) \overline{K_{3}}+(2(q-k)-1) \overline{K_{2}} & \text { for } n=4 q-k+1,0 \leq k \leq q-5 .\end{cases}$

For $n \geq 4 q+2$, a graph $G$ belongs to the family $\left\{\mathcal{H}\left(n ; T K_{n-q}\right)\right\}$ if $G$ has $n$ vertices and $\bar{G}$ is formed by $2 q+1$ nonadjacent edges (see Figure 3.1 ).

For $n=4 q-k+1$ with $q \geq 5$ and $0 \leq k \leq q-5$, a graph $G$ belongs to the family $\left\{\mathcal{H}\left(n ; T K_{n-q}\right)\right\}$ if it has $4 q-k+1$ vertices and $\bar{G}$ is formed by $k+1$ nonadjacent triangles and $2(q-k)-1$ nonadjacent edges, as Figure 3.2 shows.

In the next two sections, we will prove the following theorem.

THEOREM 3.1. $E X\left(n ; T K_{p}\right)=\left\{\mathcal{H}\left(n ; T K_{p}\right)\right\}$ for $\left\lceil\frac{2 n+6}{3}\right\rceil \leq p<n-3$. 


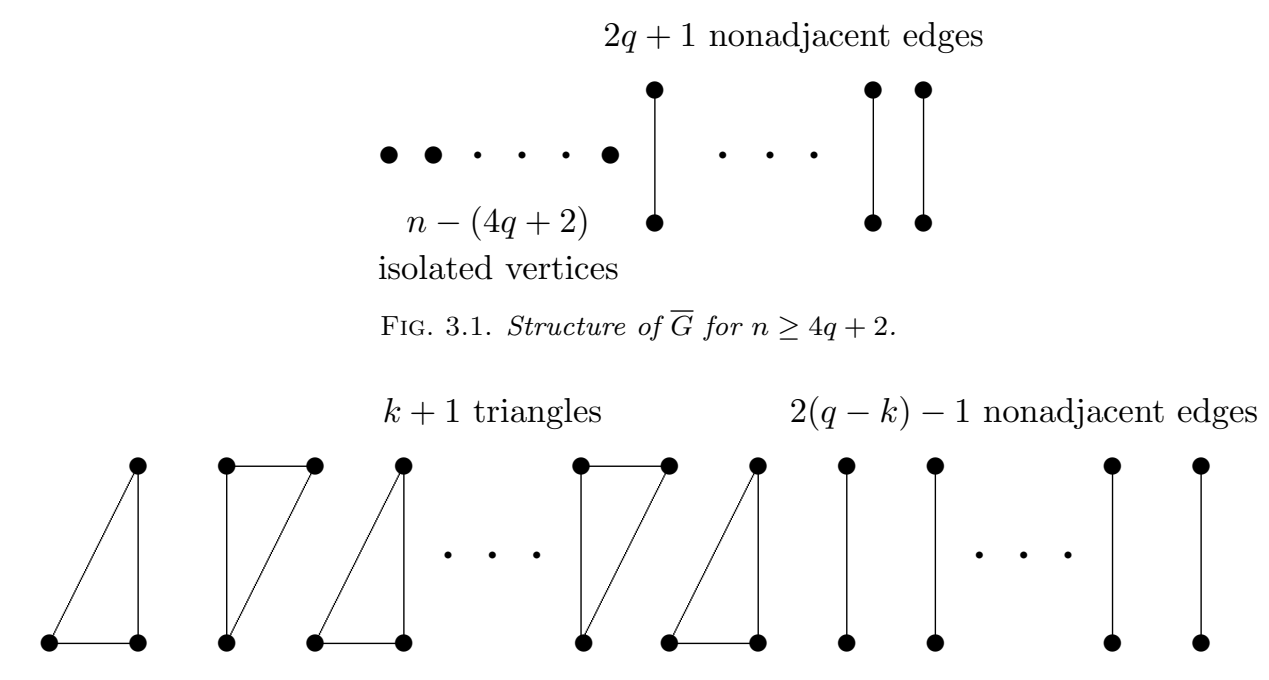

FIG. 3.2. Structure of $\bar{G}$ for $n=4 q-k+1$.

4. Case $\left\lceil\frac{3 n+2}{4}\right\rceil \leq p<n-3$. The aim of this section is to prove Theorem 3.1 when $n$ and $p$ are related by the expression $\left\lceil\frac{3 n+2}{4}\right\rceil \leq p<n-3$.

Proposition 4.1. Let $n$ and $p$ be two positive integers such that $\left\lceil\frac{3 n+2}{4}\right\rceil \leq p<$ $n-3$. It is verified that

$$
E X\left(n ; T K_{p}\right)=\left\{\mathcal{H}\left(n ; T K_{p}\right)\right\}
$$

In order to provide this proposition, we need some previous results. First, we recall the following results about the function $\operatorname{ex}\left(n ; T K_{n-q}\right)$ (see [2]).

Theorem 4.2 (see [2]). Let $n$ and $q$ be two positive integers. If $n \geq 4 q+2$, then

$$
e x\left(n ; T K_{n-q}\right)=\left(\begin{array}{c}
n \\
2
\end{array}\right)-(2 q+1) .
$$

Also, we recall that, given a graph $H$ and $v \in H$, the set of vertices adjacent to $v$ in $H$ is denoted by $\Gamma(v)$ (see [1]). Given a bipartite graph $B$ whose classes are $X$ and $Y$ with $|X| \leq|Y|$, we say that $B$ has a complete matching if there exists a set of nonadjacent edges in $B$ with cardinality $|X|$. If we need to show the existence of a complete matching in a bipartite graph, then we can use Hall's condition.

TheOrem 4.3 (see [5]). Given a bipartite graph with classes $X$ and $Y$, if $|\Gamma(A)| \geq$ $|A|$ for all $A \subseteq X$, where $\Gamma(A)=\bigcup_{v \in A} \Gamma(v)$, then there exists a complete matching.

The next result asserts that for any graph $G \in E X\left(n ; T K_{n-q}\right)$ its complement graph $\bar{G}$ is extremal for $\mathcal{C}_{q}^{q+1}$ in the sense that $\bar{G} \in \mathcal{C}_{q}^{q+1}$ and $\bar{G} \notin \mathcal{C}_{q}^{q}$.

LEMMA 4.4. Let $n$ and $q$ be two nonnegative integers with $q \geq 4$ and $n \geq 4 q+2$. For every graph $G$ from the family of graphs $E X\left(n ; T K_{n-q}\right)$, we have

$$
\bar{G} \in \mathcal{C}_{q}^{q+1}-\mathcal{C}_{q}^{q} .
$$

Proof. Let $G$ be a graph such that $G \in E X\left(n ; T K_{n-q}\right)$. The graph $G$ does not contain a subgraph homeomorphic to $K_{n-q}$, so by Theorem 4.2, we know that

$$
|E(G)|=\left(\begin{array}{c}
n \\
2
\end{array}\right)-(2 q+1)
$$


Hence, $|E(H)|=2 q+1$, where $H=\bar{G}$.

By Lemma 2.1, there exists an integer $s$ such that $H \in \mathcal{C}_{q}^{s}$. This means that there exists a subset $\left\{v_{1}, \ldots, v_{q}\right\}$ of vertices of $G$ verifying $\left|E\left(H_{q}\right)\right| \leq s$, where $H_{q}=$ $H-\left\{v_{1}, \ldots, v_{q}\right\}$. If $s \leq q+1$, then $H \in \mathcal{C}_{q}^{q+1}$. Otherwise $(s>q+1)$, let $H^{*}$ be the graph obtained from $H$ by removing one of the edges of the subgraph $H_{q}$. The graph $H^{*}$ has $n \geq 4 q+2$ vertices and $2 q$ edges, and applying Lemma 2.2 results in $H^{*} \in \mathcal{C}_{q}^{q}$. Furthermore, by the construction of the graph $H^{*}$, the set of vertices chosen to prove that $H^{*}$ belongs to the class of graphs $\mathcal{C}_{q}^{q}$ is the same as the one we chose previously in $H$; thus $\left|E\left(H_{q}\right)\right| \leq q+1$ and $H \in \mathcal{C}_{q}^{q+1}$.

Now we will prove that the number of edges of $H_{q}$ may not be equal to or less than $q$, i.e., $H \notin \mathcal{C}_{q}^{q}$. Suppose that $H \in \mathcal{C}_{q}^{q}$. This means there exists a set of vertices $\left\{v_{1}, \ldots, v_{q}\right\}$ guaranteeing this assertion. Let $e_{1}=\left(a_{1}, b_{1}\right), \ldots, e_{s}=\left(a_{s}, b_{s}\right)$ be the edges of $H_{q}$ with $1 \leq s \leq q$.

We consider the bipartite graph $B$ whose classes are $X=\left\{e_{1}, \ldots, e_{s}\right\}$ and $Y=\left\{v_{1}, \ldots, v_{q}\right\}$ such that $e_{i}$ is adjacent to $v_{j}$ in $B$ if the path $a_{i} v_{j} b_{i}$ exists in $G$. We note that if there exists a complete matching in $B$, then we have that $G$ contains a subgraph homeomorphic to $K_{n-q}$. Now Hall's condition implies the existence of a complete matching. Thus, we will prove that $|\Gamma(A)| \geq|A|$ for each $A \subseteq X$.

Let $A=\left\{e_{i}\right\}$ be a subset of $X$ with $|A|=1$ for $i \in\{1, \ldots, s\}$. If $|\Gamma(A)|=0$, then $e_{i}$ is nonadjacent to any vertex of the set $\left\{v_{q-2}, v_{q-1}, v_{q}\right\}$ in $B$. Hence, no vertex $v \in\left\{v_{q-2}, v_{q-1}, v_{q}\right\}$ is adjacent to both $a_{i}$ and $b_{i}$ in $G$. Consequently, $\delta_{H_{q-1}}\left(a_{i}\right) \geq 2$ or $\delta_{H_{q-1}}\left(b_{i}\right) \geq 2$ and, furthermore, $\delta_{H_{q-3}}\left(a_{i}\right) \geq 3$ or $\delta_{H_{q-3}}\left(b_{i}\right) \geq 3$. Thus, using property (2) of the definition of $\mathcal{C}_{q}^{q}$, we obtain that $\delta_{H_{j-1}}\left(v_{j}\right) \geq 3$ for $j=1, \ldots, q-2$ and $\delta_{H_{j-1}}\left(v_{j}\right) \geq 2$ for $j=q-1, q$. Therefore, since $s \geq 1$ we have that

$$
|E(H)| \geq 3(q-2)+2 \cdot 2+s \geq 2 q+2
$$

for $q \geq 3$. But this is not possible since $|E(H)|=2 q+1$.

We consider $A=\left\{e_{i}, e_{j}\right\} \subseteq X$ for $i, j \in\{1, \ldots, s\}$ with $i \neq j$, and we suppose $|\Gamma(A)| \leq 1$. This means that at least three vertices of the set $\left\{v_{q-3}, v_{q-2}, v_{q-1}, v_{q}\right\}$ are nonadjacent to $e_{i}$ and to $e_{j}$ in $B$. Taking into account property (2) of the definition of $\mathcal{C}_{q}^{q}$, we have that $\delta_{H_{j-1}}\left(v_{j}\right) \geq 3$ for $j=1, \ldots, q-3, \delta_{H_{j-1}}\left(v_{j}\right) \geq 2$ for $j=q-2, q-1$ and $\delta_{H_{q-1}}\left(v_{q}\right) \geq 1$ (see Figure 4.1). Hence,

$$
|E(H)| \geq 3(q-3)+2 \cdot 2+1+s \geq 2 q+2
$$

for $q \geq 4$, and this is a contradiction, as in the previous case.

Let $m$ be an integer with $3 \leq m \leq s$. Let $A$ be the set of vertices $\left\{e_{i_{1}}, \ldots, e_{i_{m}}\right\} \subseteq\left\{e_{1}, \ldots, e_{s}\right\}$ with $i_{1}<i_{2}<\cdots<i_{m}$. If $|\Gamma(A)| \leq m-1$, then there

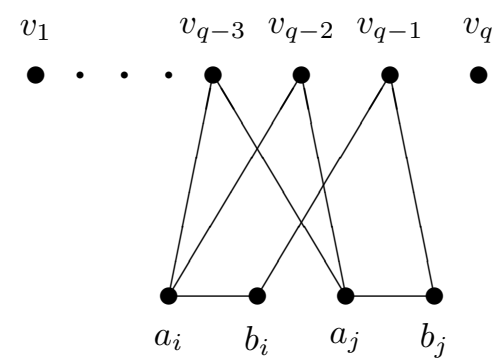

FIG. 4.1. Possible structure of $H$ for the most unfavorable case for $A=\left\{e_{i}, e_{j}\right\}$. 


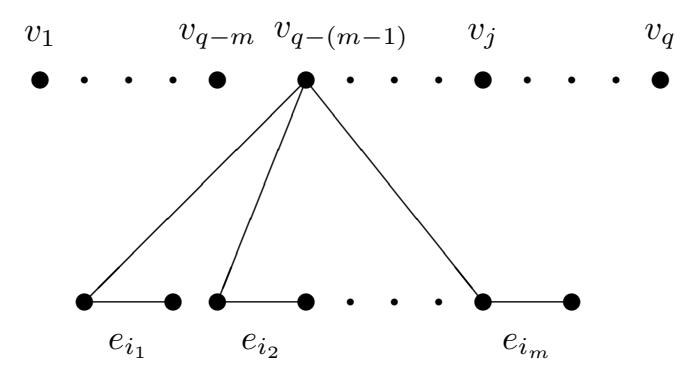

FIG. 4.2. Possible structure of $H$ for the most unfavorable case for $3 \leq m \leq s$.

exists $i \in\{q-(m-1), \ldots, q\}$ in such a way that $v_{i}$ is not adjacent to any vertex of the set $A$ in the graph $B$. By applying condition (2) of the definition of $\mathcal{C}_{q}^{q}$, we obtain that $\delta_{H_{q-m}}\left(v_{q-(m-1)}\right) \geq m$ and, therefore, $\delta_{H_{j-1}}\left(v_{j}\right) \geq m$ for $1 \leq j \leq q-(m-1)$ (see Figure 4.2). Furthermore, $\delta_{H_{j-1}}\left(v_{j}\right) \geq 1$ for $q-(m-2) \leq j \leq q$ and $\left|E\left(H_{q}\right)\right|=s \geq m$. Consequently,

$$
\begin{aligned}
|E(H)| & \geq m(q-(m-1))+m-1+s \\
& \geq m q-m^{2}+3 m-1 .
\end{aligned}
$$

Since $E(H)=2 q+1$, we have that $2 q+1 \geq m q-m^{2}+3 m-1$ and, therefore, $q \leq \frac{m^{2}-3 m+2}{m-2} \leq m-1<m \leq s$, but this is not possible. Therefore, $|\Gamma(A)| \geq|A|$ for each $A \subseteq X$. Thus, by Hall's condition, there exists a complete matching in $B$ and, thereby, the graph $G$ contains a subgraph homeomorphic to $K_{n-q}$. This is not possible, and the result follows.

Now we can prove Proposition 4.1.

Proof of Proposition 4.1. It is equivalent to prove that

$$
E X\left(n ; T K_{n-q}\right)=\left\{\mathcal{H}\left(n ; T K_{n-q}\right)\right\}
$$

for $q \geq 4$ and $n \geq 4 q+2$.

Let $G$ be a graph belonging to $\left\{\mathcal{H}\left(n ; T K_{n-q}\right)\right\}$ with $n \geq 4 q+2$. It is easy to check that $G$ does not contain a subgraph homeomorphic to $K_{n-q}$. Furthermore, by denoting $|E(G)|$ as the number of edges of $G$, we have that

$$
|E(G)|=\operatorname{ex}\left(n ; T K_{n-q}\right)=\left(\begin{array}{c}
n \\
2
\end{array}\right)-(2 q+1) .
$$

Thus, by Theorem 4.2, $G$ is maximal on edges and

$$
\left\{\mathcal{H}\left(n ; T K_{n-q}\right)\right\} \subseteq E X\left(n ; T K_{n-q}\right) .
$$

In order to prove that $E X\left(n ; T K_{n-q}\right) \subseteq\left\{\mathcal{H}\left(n ; T K_{n-q}\right)\right\}$, let $G$ be a graph belonging to $E X\left(n ; T K_{n-q}\right)$, and we set $H=\bar{G}$. By Theorem 4.2 we have that $|E(H)|=2 q+1$. By Lemma 2.1, we know there exists $s$ such that $H \in \mathcal{C}_{q}^{s}$. Let $\left\{v_{1}, \ldots, v_{q}\right\}$ be a set of $q$ vertices guaranteeing this property. We know that there exists a vertex $v \in H_{q}$ such that $\delta_{H_{q}}(v) \geq 1$, because otherwise $H_{q}$ is empty and $H \in \mathcal{C}_{q}^{q}$. But this is not possible because, by Lemma 4.4, we know that $H \notin \mathcal{C}_{q}^{q}$. If $\delta\left(v_{1}\right) \geq 2$, then $\left|E\left(H_{q}\right)\right| \leq 2 q+1-(2+q-1)=q$ and therefore $H \in \mathcal{C}_{q}^{q}$, a contradiction. Therefore, $\delta\left(v_{1}\right) \leq 1$.

Thus, as $v_{1}$ is the vertex of maximum degree in $H$, we have that $\delta(v) \leq 1$ for all $v \in H$, and then the graph $H$ is formed by $2 q+1$ nonadjacent edges. Therefore, the result follows. 
5. Case $\left\lceil\frac{2 n+6}{3}\right\rceil \leq p<\left\lceil\frac{3 n+2}{4}\right\rceil$. In this section, we will characterize the family of extremal graphs $E X\left(n ; T K_{n-q}\right)$ for $n=4 q-k+1$ with $0 \leq k \leq q-5$ in such a way that we will show that $E X\left(n ; T K_{n-q}\right)=\left\{\mathcal{H}\left(n ; T K_{n-q}\right)\right\}$, applying techniques based on the same ideas as in the previous section.

THEOREM 5.1. Let $n$ and $p$ be two positive integers with $\left\lceil\frac{2 n+6}{3}\right\rceil \leq p<\left\lceil\frac{3 n+2}{4}\right\rceil$. Then

$$
E X\left(n ; T K_{p}\right)=\left\{\mathcal{H}\left(n ; T K_{p}\right)\right\}
$$

In order to prove this result, we also need to recall some results about the function $e x\left(n ; T K_{n-q}\right)$ (see [2]).

LEMma 5.2 (see [2]). Let $k$ be a nonnegative integer and $H$ be a graph with maximum degree 2 and at least $3 k+1$ vertices of maximum degree. Then there exist at least $k+1$ nonadjacent vertices with degree 2 .

TheOREm 5.3 (see [2]). Let $n, k$, and $q$ be three nonnegative integers with $0 \leq$ $k \leq q-4$ and $n=4 q-k+1$. It is verified that

$$
\operatorname{ex}\left(n ; T K_{n-q}\right)=\left(\begin{array}{c}
n \\
2
\end{array}\right)-(2 q+k+2) .
$$

Now we will show, as in Lemma 4.4, that if $G \in E X\left(n ; T K_{n-q}\right)$ with $n=4 q-k+1$, then $\bar{G} \in \mathcal{C}_{q}^{q+1}$ but $\bar{G} \notin \mathcal{C}_{q}^{q}$.

LEMMA 5.4. Let $k$, $n$, and $q$ be three nonnegative integers such that $q \geq 5$, $0 \leq k \leq q-5$, and $n=4 q-k+1$. If $G \in E X\left(n ; T K_{n-q}\right)$, then

$$
\bar{G} \in \mathcal{C}_{q}^{q+1}-\mathcal{C}_{q}^{q} .
$$

Proof. Let $G$ be a graph belonging to $E X\left(n ; T K_{n-q}\right)$. This graph does not contain a graph homeomorphic to $K_{n-q}$, and by Theorem 5.3 we know that

$$
|E(G)|=\left(\begin{array}{c}
n \\
2
\end{array}\right)-(2 q+k+2) .
$$

Thus, $H=\bar{G}$ has $2 q+k+2$ edges.

Let $H^{*}$ be the graph obtained from $H$ by removing one edge, similar to what we have done in Lemma 4.4. Since $H^{*}$ is a graph formed by $4 q-k+1$ vertices and $2 q+k+1$ edges, then applying Lemma 2.3 yields $H^{*} \in \mathcal{C}_{q}^{q}$, and then

$$
H \in \mathcal{C}_{q}^{q+1} .
$$

Now we will show that $H \notin \mathcal{C}_{q}^{q}$. To the contrary, suppose $H \in \mathcal{C}_{q}^{q}$ and let $\left\{v_{1}, \ldots, v_{q}\right\}$ be a set of vertices of $H$ guaranteeing that $H \in \mathcal{C}_{q}^{q}$. Let $e_{1}=\left(a_{1}, b_{1}\right), \ldots$, $e_{s}=\left(a_{s}, b_{s}\right)$ be the edges of $H_{q}$ with $s \leq q$. We consider the bipartite graph $B$ constructed as in Lemma 4.4, i.e., the graph whose classes are $X=\left\{e_{1}, \ldots, e_{s}\right\}$ and $Y=\left\{v_{1}, \ldots, v_{q}\right\}$ in such a way that $e_{i}$ is adjacent to $v_{j}$ if the path $a_{i} v_{j} b_{i}$ exists in the graph $G$. In this case, if we show the existence of a complete matching in $B$, then we would have that $G$ contains a subgraph homeomorphic to $K_{n-q}$. Therefore, we will show that $|\Gamma(A)| \geq|A|$ for each $A \subseteq X$.

If $|A|=m=1$, by reasoning as in the proof of Lemma 4.4, we have that

$$
|E(H)| \geq 3(q-2)+4+s=3 q+s-2 \geq 3 q-1 .
$$


Since $k \leq q-4$, it is verified that $3 q-1 \geq 2 q+k+4-1>2 q+k+2$, but this is not possible.

For $m=2$, by considering as done previously, we have that

$$
|E(H)| \geq 3(q-3)+4+1+s=3 q-4+s \geq 3 q-2 .
$$

Taking into account that $k \leq q-5$, it is verified that $|E(H)|>2 q+k+2$, and this is a contradiction.

We consider $m=3$. Let $A=\left\{e_{i_{1}}, e_{i_{2}}, e_{i_{3}}\right\}$ be a subset of vertices of $X$ with $1 \leq i_{1}<i_{2}<i_{3} \leq s$. If $|\Gamma(A)| \leq 2$, then there exists $i \in\{q-2, \ldots, q\}$ in such a way that $v_{i}$ is not adjacent to any vertex of the set $A$ in the graph $B$. Hence, by applying property (2) of the definition of $\mathcal{C}_{q}^{q}$, we have that $\delta_{H_{q-3}}\left(v_{q-2}\right) \geq 3$. Thus,

$$
|E(H)| \geq 3(q-2)+2+s \geq 3 q-1>2 q+k+2
$$

since $k \leq q-4$.

In general, if $4 \leq m \leq s$, then we consider $A$ as the set of vertices $\left\{e_{i_{1}}, \ldots, e_{i_{m}}\right\} \subseteq\left\{e_{1}, \ldots, e_{s}\right\}$ with $i_{1}<i_{2}<\cdots<i_{m}$. If $|\Gamma(A)| \leq m-1$, then there exists $i \in\{q-(m-1), \ldots, q\}$ in such a way that $v_{i}$ is not adjacent to any vertex of the set $A$ in the graph $B$. Hence, as in the proof of Lemma 4.4, we have that $\delta_{H_{q-m}}\left(v_{q-(m-1)}\right) \geq m$ and, therefore,

$$
|E(H)| \geq m(q-(m-1))+m-1+s \geq m q-m^{2}+3 m-1 .
$$

But $|E(H)|=2 q+k+2 \leq 3 q-3$ for $k \leq q-5$. Thus, $3 q-3 \geq m q-m^{2}+3 m-1$ and, thereby, $q \leq m-\frac{2}{m-3}<m$, but this is not possible.

Thus, using Hall's condition, there exists a complete matching in $B$, and consequently, $G$ contains a subgraph homeomorphic to $K_{n-q}$, but this is not possible. Hence, $H \notin \mathcal{C}_{q}^{q}$ and the result follows.

The next result is devoted to proving the existence of nonadjacent triangles in graphs with maximum degree 2 and the prescribed number of vertices of maximum degree.

LEMMA 5.5. Let $r$ be a nonnegative integer, and let $H$ be a graph with maximum degree 2. If $H$ has $3 r+3$ vertices of degree 2 and $r+1$ of them form an independent set, then $H$ contains $r+1$ nonadjacent triangles.

Proof. We apply induction on $r$. For $r=0$ the result is obvious, because the triangle is the unique graph formed by 3 vertices of degree 2 and all of them are adjacent among themselves.

Now suppose that $r+1 \geq 2$ and the result holds for $r$. Let $H$ be a graph with $3(r+1)+3=3(r+2)$ vertices of degree 2 , and let $w_{1}, \ldots, w_{r+2}$ be $r+2$ nonadjacent vertices of $H$.

If there exist $i, j \in\{1, \ldots, r+2\}$ with $i \neq j$ such that $\Gamma\left(w_{i}\right) \cap \Gamma\left(w_{j}\right) \neq \emptyset$, then $\left|\bigcup_{k=1}^{r+2}\left\{\Gamma\left(w_{k}\right) \cup w_{k}\right\}\right|<3(r+2)$. Thus, there exists $w \in H$ with degree 2 nonadjacent to $w_{i}$ for all $i$. Hence, $\left\{w, w_{1}, \ldots, w_{r+2}\right\}$ is a set of $r+3$ nonadjacent vertices of degree 2 , but this is a contradiction. Therefore, $\Gamma\left(w_{i}\right) \cap \Gamma\left(w_{j}\right)=\emptyset$ for all $i \neq j$. Furthermore, if $w \in H$ is adjacent to any $w_{i}$ for $i \in\{1, \ldots, r+2\}$, then $w$ has degree 2; otherwise, since the number of vertices of degree 2 is $3(r+2)$, there exists $v \in H$ with degree 2 nonadjacent to $w_{i}$ for all $i$, and we have seen above that this is not possible.

Now, let $a$ and $b$ be the vertices adjacent to $w_{r+2}$. If the edge $(a, b)$ does not belong to $H$, we have that $\left\{w_{1}, \ldots, w_{r+1}, a, b\right\}$ is a set of $r+3$ nonadjacent vertices of degree 2. Thus, the vertices $w_{1}, a$, and $b$ form a triangle. 
Denote by $H^{*}$ the graph obtained from $H$, removing the previous triangle. Therefore, $H^{*}$ is a graph with $3 r+3$ vertices of degree 2 , and $r+1$ of them are nonadjacent; by induction hypothesis, $H^{*}$ contains $r+1$ nonadjacent triangles. Thus, $H$ contains $r+2$ nonadjacent triangles.

To finish this section, we give the proof of Theorem 5.1, using the previous results.

Proof of Theorem 5.1. It is equivalent to show that

$$
E X\left(n ; T K_{n-q}\right)=\left\{\mathcal{H}\left(n ; T K_{n-q}\right)\right\}
$$

for $n=4 q-k+1$ with $q \geq 5,0 \leq k \leq q-5$.

Let $G$ be a graph belonging to the set $\left\{\mathcal{H}\left(n ; T K_{n-q}\right)\right\}$. By checking the structure of this graph $G$, it is easy to prove that $G$ does not contain a subgraph homeomorphic to $K_{n-q}$. Since $|E(G)|=\operatorname{ex}\left(n ; T K_{n-q}\right)=\left(\begin{array}{c}n \\ 2\end{array}\right)-(2 q+k+2)$, we have that $G \in E X\left(n ; T K_{n-q}\right)$.

In order to show that $E X\left(n ; T K_{n-q}\right) \subseteq\left\{\mathcal{H}\left(n ; T K_{n-q}\right)\right\}$, let $G$ be a graph belonging to $E X\left(n ; T K_{n-q}\right)$. We denote by $H=\bar{G}$. By Theorem $5.3,|E(H)|=2 q+k+2$. First, we will prove that $\Delta(H) \leq 2$. Suppose the contrary, that $\Delta(H) \geq 3$.

By applying Lemma 5.4, we have $H \in \mathcal{C}_{q}^{q+1}-\mathcal{C}_{q}^{q}$. Hence, there exists a subset of vertices $\left\{v_{1}, \ldots, v_{q}\right\}$ of $H$ guaranteeing this property. Furthermore, $\left|E\left(H_{q}\right)\right|=q+1$. We claim there exists $j \in\{1, \ldots, q\}$ such that $\Delta\left(H_{j-1}\right) \geq 3$ and $\Delta\left(H_{j}\right) \leq 2$, because otherwise we have $\delta_{H_{i-1}}\left(v_{i}\right) \geq 3$ for each $1 \leq i \leq q$, and

$$
|E(H)| \geq 3 q+(q+1)>2 q+k+2,
$$

but this is not possible. Now we distinguish the cases $j \geq k+1$ and $j \leq k$.

For $j \geq k+1$, we consider the fact that $\Delta\left(H_{j-1}\right) \geq 3$ and $\Delta\left(H_{j}\right) \leq 2$. Taking into account property (2) of the definition of $\mathcal{C}_{q}^{q+1}$ and $\left|E\left(H_{q}\right)\right|>0$, we have $\delta_{H_{i-1}}\left(v_{i}\right) \geq 3$ for $1 \leq i \leq j$ and $\delta_{H_{i-1}}\left(v_{i}\right) \geq 1$ for $j+1 \leq i \leq q$. Hence,

$$
\left|E\left(H_{q}\right)\right| \leq 2 q+k+2-(3 j+(q-j)) \leq q-j+1 \leq q .
$$

But this is not possible since $\left|E\left(H_{q}\right)\right|=q+1$.

For $j \leq k$, we have that $\delta_{H_{i-1}}\left(v_{i}\right) \geq 3$ for $1 \leq i \leq j$. If $\Delta\left(H_{k}\right) \leq 1$, then $2\left|E\left(H_{k}\right)\right| \leq\left|V\left(H_{k}\right)\right|$ and

$$
4 q-2 k+1=\left|V\left(H_{k}\right)\right| \geq 2\left|E\left(H_{k}\right)\right| \geq 2(q-k+q+1)=4 q-2 k+2,
$$

and this is a contradiction. Thus, $\Delta\left(H_{k}\right)=2$ and $\delta_{H_{i-1}}\left(v_{i}\right) \geq 2$ for $j+1 \leq i \leq k$. Hence,

$$
\left|E\left(H_{q}\right)\right| \leq 2 q+k+2-(3 j+2(k-j+1)+(q-k+1))=q-j+1 \leq q,
$$

and this not possible. Thus, $\Delta(H) \leq 2$.

Since $2|E(H)|>|V(H)|$, we have $\Delta(H) \geq 2$ and, consequently, $\Delta(H)=2$.

Next we are going to study the structure of $H$. On the one hand, if $H$ has at least $3(k+1)+1$ vertices of degree 2 , then by Lemma 5.2 we have that $k+2$ of those vertices $\left\{w_{1}, \ldots, w_{k+2}\right\}$ are nonadjacent. Let $w_{k+3}, \ldots, w_{q}$ be $q-(k+2)$ vertices of $H$ such that the set $\left\{w_{1}, \ldots, w_{k+2}, w_{k+3}, \ldots, w_{q}\right\}$ verifies properties (1) and (2) of the definition of $\mathcal{C}_{q}^{s}$. For this set of vertices, we have that

$$
\left|E\left(H_{q}\right)\right| \leq 2 q+k+2-(2(k+2)+q-(k+2))=q,
$$


and therefore, $H \in \mathcal{C}_{q}^{q}$, a contradiction. Thus, $H$ has at most $3 k+3$ vertices of degree 2 . On the other hand, if we denote by $n_{i}$ the number of vertices of degree $i$ in $H$, we have that

$$
\left.\begin{array}{rl}
2 n_{2}+n_{1} & =2(2 q+k+2) \\
n_{2}+n_{1}+n_{0} & =4 q-k+1
\end{array}\right\}
$$

Thus, $n_{2}=3 k+3+n_{0} \geq 3 k+3$ and the number of vertices of degree 2 in $H$ is $n_{2}=3 k+3$.

Furthermore, as we have shown previously, $H$ may not have $k+2$ nonadjacent vertices of degree 2 . Since $H$ has $3 k+3 \geq 3 k+1$ vertices of degree 2 , by Lemma 5.2 we have that $H$ has at least $k+1$ nonadjacent vertices. Hence, $H$ has maximum degree 2 and $3 k+3$ vertices of degree 2 , and $k+1$ of them are nonadjacent. Therefore, by applying Lemma 5.5, $H$ contains $k+1$ nonadjacent triangles. Additionally, $n_{0}=0$, $n_{1}=4 q-4 k-2$, and the result follows.

Acknowledgment. The authors thank the referees for their helpful comments and suggestions.

\section{REFERENCES}

[1] B. Bollobas, Extremal Graph Theory, Academic Press, London, 1978.

[2] M. Cera, A. Diánez AND A. MÁrquez, The size of a graph without topological complete subgraphs, SIAM J. Discrete Math., 13 (2000), pp. 295-301.

[3] G. A. DiRAC, In abstrakten Graphen vorhandene vollständige 4-Graphenund ihre Unterteilungen, Math. Nachr., 22 (1960), pp. 61-85.

[4] G. A. Dirac, Homeomorphism theorem for Graphs, Math. Ann., 153 (1964), pp. 69-80.

[5] P. Hall, On representatives of subsets, J. London Math. Soc., 10 (1935), pp. 26-30.

[6] W. Mader, Homomorphieegenshaften und mittlere Kantendichte von Graphen, Math. Ann., 174 (1967), pp. 265-268.

[7] W. MADER, Hinreichende Bedingungen für die Existenz von Teilgraphen, diezu einem vollständigen Graphen Homömorph sind, Math. Nachr., 53 (1972), pp. 145-150.

[8] W. MAder, Graphs without a Subdivision of $K_{5}$ of Maximum Size, preprint, 1998.

[9] W. MAder, $3 n-5$ edges do force a subdivision of $K_{5}$, Combinatorica, 18 (1998), pp. 569-595.

[10] C. Thomassen, Some homomorphism properties of graphs, Math. Nachr., 64 (1974), pp. 119133. 